\title{
Research on the Texture-mapping of the Three-dimensional Urban Roadway Simulation of Virtual Battlefield
}

\author{
Hou Runfeng, Chu Shili, Li Chenhui, Shi Lei, Qi Genhua \\ Air Defense Forces Command Academy \\ Zhengzhou 450052, China
}

\begin{abstract}
Building virtual battlefield is an important subject in military simulation. In order to get realistic appearance in building roadway of battlefield by using 3D simulation technology, it's natural to do some complex calculations for the surface of the roadway, involving patterns with certain colours and textures. Usually, the surface of roadway with single colour can be simulated by direct colouration. The surface involving complicated patterns with colours and textures need to use texture-mapping. In this paper, it's demonstrated that, not only the complexity of the calculation for the surface can be simplified, but also the improvement of the efficiency and quality for the 3D simulation of the roadway can be achieved, by choosing proper texture pattern and appropriate texture mapping. Thereby, a satisfactory roadway constructed with 3D simulation technology appears.
\end{abstract}

Keywords-Virtual battlefield, 3D simulation, Roadway, Texture mapping

\section{INTRODUCTION}

The research of the roadway simulation technology becomes important in the urban operation. In order to bring intuitive feelings of battlefield, the 3D simulation environment should display the appearance of the roadway realistically. Because the complexity and diversity of the roadway appearance, the simulation of some features is not real if only by ways of the coloration painting. However, if we can make use of the texture-mapping technology rationally, the simulation of the complex roadway appearance will become simple and realistic. The calculation will be also simplified meanwhile. For the purpose of improving the display quality and efficiency, it is needs to find out which features need to use texturemapping and which only need simple coloration paintings. On the occasion that using texture-mapping, the chosen of texture images and mapping coordinates will impact the simulation effect.

In this paper, the technology of texture-mapping and the proper texture mapping rules are used to achieve the simulation of the roadway surface slope. Instead of simple coloration painting for the channelization islands, the technology of texture-mapping can simplify the calculation. We choose appropriate texture image to improve the sky box technology.

\section{THE ROADWAY SLOPE REALITY OF THE VIRTUAL}

\section{BATTLEFIELD}

\section{A. Proposing and analyzing}

When the roadway is builded, between surface and bed of the roadway is slope and its surface is usually covered with grasses and trees. In 3D simulation, the slope should be simulated by texture mapping.

The surface of a slope $G$ is a ruled surface, starting from $R(t) t \in[0, L]$, the boundary curve of the roadway, to the bed in the direction of outer normal. $t$ is an arc-length parameter and $L$

is the total arc-length of $R(t)$. Let $Q(t)$ be the external boundary of $G$ owning to the $R(t)$ 's extending. So the slope surface could be expressed as:

$$
G(s, t)=(1-s) R(t)+s Q(t) \quad(s, t) \in[0,1] \times[0, L]
$$

$G(s, t)$ is a straight-line group composition which connects the corresponding points of both the boundary $R(t)$ and $Q(t)$.

A simple way to determine the texture-mapping is to use $(x, y)$, the projection on the earth plane of the 3D coordinates $(x, y, z)$ of the point $P$, as texture-mapping coordinates. As shown in Figure 1, with changes of the roadway extension, the texture pattern becomes slanted, which affects the realistic of the slope. Hence, a reasonable texture mapping to improve the simulation is needed.

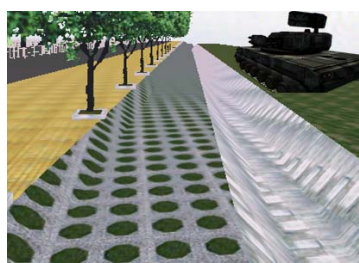

Figure 1. The view using the projection on the earth as the texture

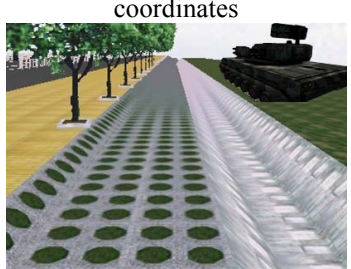

Figure 2.The view using the texture coordinates specified 


\section{B. The realization}

The above problem will be discussed as follows. The texture-mapping coordinates will be changed, which will improves the effect of texture-mapping greatly.

1) Determine the texture coordinates and let $R(t)(t \in[0, L])$ map onto the left boundary of the texture image.

Assume that there is a texture mapping:

$$
f_{1}: \quad A_{1} \rightarrow B_{1}
$$

In (2), the domain $A_{1}=\{R(t) \mid t \in[0, L]\}$, and $f_{1}$ satisfies

$f_{1}(R(t))=(0, t)$. So, the texture coordinates of

$R(t)(t \in[0, L])$ is $(0, t)$ by $f_{1}$. From (3), the inner

boundary of $G$ maps onto the left boundary of the texture image, the first texture coordinate of a point on $R(t)(t \in[0, L])$ is 0 .

2) Determine the texture coordinates and let the texture coordinates extend linearly when the surface extends from $R(t)(t \in[0, L])$ to $Q(t)(t \in[0, L])$.

Let $\forall P=G(s, t)$, and $l=|P R(t)|$, which means the total length of the line segment $P R(t)$.

Assume that there is a texture mapping:

$$
f_{2}: \quad A_{2} \rightarrow B_{2}
$$

And the domain is

$$
\begin{gathered}
A_{2}=\{P \mid P=G(s, t)=(1-s) R(t)+s Q(t) \\
(s, t) \in[0,1] \times[0, L]\}
\end{gathered}
$$

$f_{2}$ satisfies $f_{2}(P)=(l, t)$, where $l$ is the distance from $P$ to the inner boundary of $G(s, t) ;(l, t)$ is the texture coordinate of $P$. By (5), the first texture coordinate of $P$ is $l$. The texture coordinates on the surface $G(s, t)$ extend from the inner boundary to the outer boundary linearly.

The view using the coordinates above is shown in Figure 2. The similar method could be used to improve the quality and effect in carriageway, green strip, sidewalk, and so on of the virtual battlefield.

\section{THE CHANNELIZATION ISLAND REALITY OF THE}

\section{VIRTUAL BATTLEFIELD}

\section{A. Proposing and analyzing}

As shown in Figure 3, let $S$ be the surface of a channelization island. The boundary of $\mathrm{S}$ is always a curve. After marking on $S$, the marking regions are curved surfaces. Let $s$ be one of the marking surfaces, with vertexes $p_{0}, p_{1}, p_{2}, p_{3}$ Usually, $s$ is a known width strip intercepted by $S$, and its boundaries $\widehat{p_{0} p_{1}}, \widehat{p_{2} p_{3}}$ locate on the boundary of $S$.In 3D simulation, the boundary of $S$ is always circular arc, cyclotron line and straight line, so it should be approximated by linear interpolation. In order to improve the showing efficiency, the interpolation step is always dynamic. Therefore the boundary of the surface $S$, obtained by interpolation, is dynamic. $\widetilde{p_{0} p_{1}}$ and $\widetilde{p_{2} p_{3}}$ are dynamic, too. To make sure that $\widehat{p_{0} p_{1}}$ and $\widehat{p_{2} p_{3}}$ are not in or out of $S$, they should be obtained temporarily, and those make the calculations of the channelization island become very complicated.

We could reasonably map the channelization island onto the surface $S$ by choosing a suitable texture image and texture mapping coordinates. Then the complexity of the calculation can be avoided, and $\overparen{p_{0} p_{1}}$ and $\overparen{p_{2} p_{3}}$ are just on the boundary of the surface $S$.

\section{B. The realization}

According to the shape of the marking on the channelization island, the image in Figure 4 is the texture image of the channelization island that shown in Figure 3. As $S$ is not convex, the tessellation in the OpenGL is used to achieve the simulation. So we should just know the texture coordinates of every point on the boundary of $S$.

The texture coordinates are determined as the following methods:

1) Establish appropriate texture coordinates according to the texture image and the extension of the channelization island marking.

In the texture coordinates of the channelization island, the origin is usually a vertex of $S$ and the quadrant bisector specifies the direction of the marking sign. By determining the origin of texture coordinates on $S$, the lower left quarter of the texture image should be mapped onto a yielding point of the surface boundary (a vertex instead of that if the surface is not smooth). Through the rotation of coordinates, the marking on the texture image could be accorded to the extension of that on the channelization island.

When using the Cartesian coordinates for texture mapping, the origin $P_{0}$ and the direction vector of the marking should be given as the raw data of the channelization island. Let $P_{0}$ be the origin, the x-axial and $y$-axial can be obtained by anti-clockwise and clockwise rotating the marking vector $45^{\circ}$. The coordinates on the surface of $S$ are shown in Figure 5. If $P_{1}$ is a point on the positive direction of the $\mathrm{x}$-axial, then the unit vector of the $\mathrm{x}$-axial is

$$
u=\frac{\overrightarrow{P_{0} P_{1}}}{\left|\overrightarrow{P_{0} P_{1}}\right|} \triangleq(x, y)
$$

The unit vector of the $y$-axial is $v=(-y, x)$ accordingly.

2) Get the texture coordinates of the points on the boundary of $S$

Let $\left(x_{t e x}, y_{t e x}\right)$ be the texture coordinates of a point $P$ on $S$, then

$$
x_{\text {tex }}=\overrightarrow{P_{0} P} \cdot v . y_{\text {tex }}=\overrightarrow{P_{0} P} \cdot u
$$


Let $l$ be the total width of the marking and the interval of the marking in the texture image, and $L$ be the real width channelization island. What we should do is to compress $L$ into $l$ from the system of channelization island coordinates to texture coordinates.

Assume:

$$
x_{\text {tex }}{ }^{\prime}=x_{\text {tex }} * \frac{l}{L} y_{\text {tex }}{ }^{\prime}=y_{\text {tex }} * \frac{l}{L}
$$

Only some translations and rotations have been done in (6), so the size of the channelization island has not changed. That is to say the total width of the marking and the interval of the marking is also $L$ in the texture coordinates of (6). After the transform in (7), the width above is compressed from $L$ to $l$. The texture coordinate of a point in $S$ is $\left(x_{\text {tex }}{ }^{\prime}, y_{\text {tex }}{ }^{\prime}\right)$.

By choosing right coordinates and translating the texture coordinates, the extension and the width of the marking in the process of texture-mapping do not be changed, as shown in figure 6. By using texture-mapping, the texture border completely is coincided with the surface boundary and the marking could not be passed or into the surface $S$. The calculation is simplified.

\section{IV. . CONCLUSION}

In the $3 \mathrm{D}$ dynamic simulation of the virtual battlefield, the stretched and distorted about the texture of the roadway

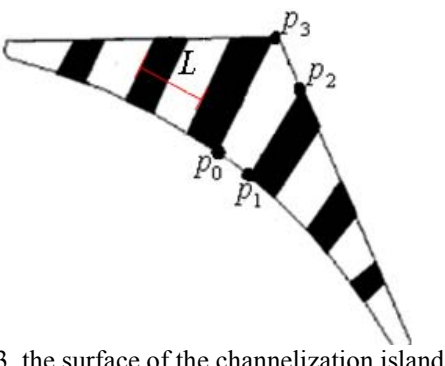

Figure 3. the surface of the channelization island

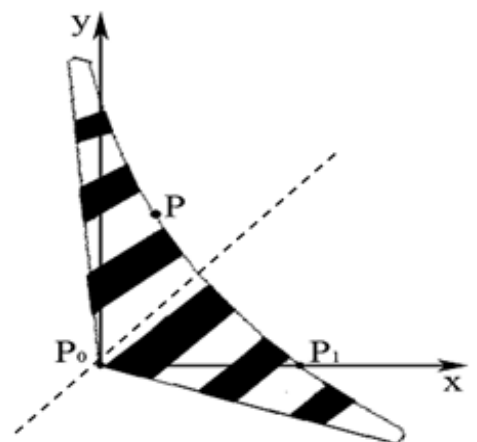

Figure 5. The coordinates system on the texture coordinates of the channelization island surfaces is improved. An overall view about a urban roadway of virtual battlefield is shown in figure 7 .

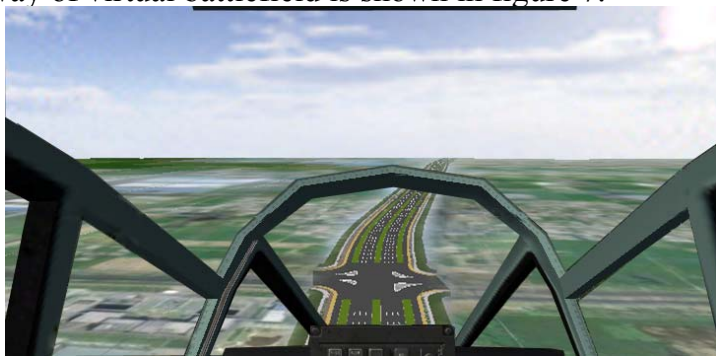

Figure 7. The overall of the 3D simulation of the urban roadway

\section{REFERENCES}

[1] Dava Shreiner, Mason Woo, Jackie Neider, Tom Davis, "OpenGL Programming Guide", Forth Edition, 2007

[2] Edward Angel, Interactive Computer Graphics: "A top-Down Approach Using OpenGL", Fifth Edison, Addison Wesley, Mar 24, 2008

[3] Chanyang Liu, Xiaoping Zhou, "Computer Graphics-The Calculation And Display Principle For The Graphics", Xidian University press, 2005

[4] Richard S Wright, Benjamin Lipichak, "OpenGL SuperBible”, Forth Edition,Addison Wesley,2007.6

[5] Ferguson.D.R.(1986), "Construction of curves and surfaces using numerical optimization techuniques", Computer-Aided Design 18,15-

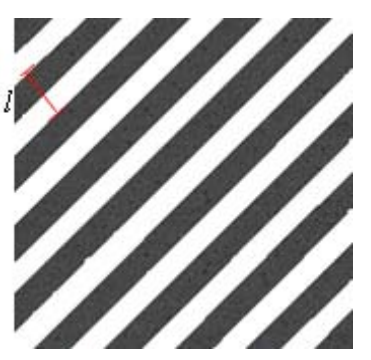

Figure 4. the texture image of the channelization island

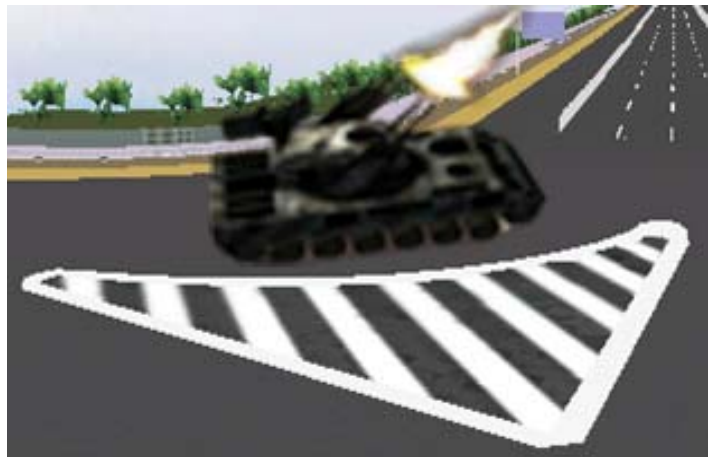

Figure 6 . the simulation of the channelization island using texture mapping 